\title{
Feasibility of supercontinuum sources for use in glucose sensing by absorption spectroscopy (Erratum)
}

Silje Skeide Fuglerud, Karolina Milenko, Reinhold Ellingsen, Astrid Aksnes, Dag Roar Hjelme

Silje Skeide Fuglerud, Karolina Milenko, Reinhold Ellingsen, Astrid Aksnes, Dag Roar Hjelme, "Feasibility of supercontinuum sources for use in glucose sensing by absorption spectroscopy (Erratum)," Proc. SPIE 11073, Clinical and Preclinical Optical Diagnostics II, 1107323 (3 February 2022); doi: $10.1117 / 12.2632461$ 


\title{
Feasibility of supercontinuum sources for use in glucose sensing by absorption spectroscopy (Erratum)
}

\author{
S. S. Fugleruda,b, K. Milenkoa, R. Ellingsena, A. Aksnes ${ }^{a}$, and D. R. Hjelmea \\ aDept. of Electronic Systems, NTNU, Trondheim, Norway \\ bSt. Olavs University Hospital, Trondheim, Norway
}

\begin{abstract}
Proceedings Volume 11073, Clinical and Preclinical Optical Diagnostics II; 110730E (2019) https://doi.org/10.1117/12.2524005

Event: European Conferences on Biomedical Optics, 2019, Munich, Germany
\end{abstract}

A revised version of this manuscript was published on 4 February 2022. Details of the revision are provided in the text that accompanies this Erratum. The original paper has been updated.

In the original article, the absorptivity of water used to calculate the change in intensity for small glucose concentrations was recorded at $22{ }^{\circ} \mathrm{C},{ }^{2}$ whereas the glucose values were recorded at $37^{\circ} \mathrm{C} .{ }^{1}$ Figure 1 was therefore incorrect. Instead, values for water absorptivity from Amerov et al. ${ }^{1}$ recorded at $37^{\circ} \mathrm{C}$ were used in this erratum. The corrected absorptivity of water and the molar absorptivity of glucose at $37^{\circ} \mathrm{C}$ both based on Amerov et al. ${ }^{1}$ are plotted in Fig. 1(a). The resulting difference for a $1 \mathrm{~mm}$ change of glucose for $1 \mathrm{~mm}$ path length is plotted in Figure 1(b). The change in the relative intensity $I / I_{0}$ can be found from Beer's law presented in Eq. 1 in the original draft. We would like to note that since Amerov et al. defined Eq. 1 by $\log _{10}$ and not $\ln$, the expected change in intensity must be calculated with the conversion factor

$$
\frac{\epsilon_{\mathrm{meas}} c}{\log _{10}(e)}=\frac{\epsilon_{\mathrm{meas}} c}{0.4343}=\ln \left(\frac{I}{I_{0}}\right)
$$

(a)

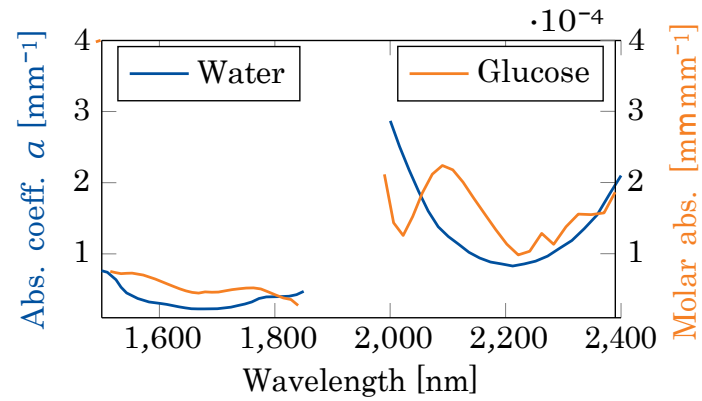

(b)

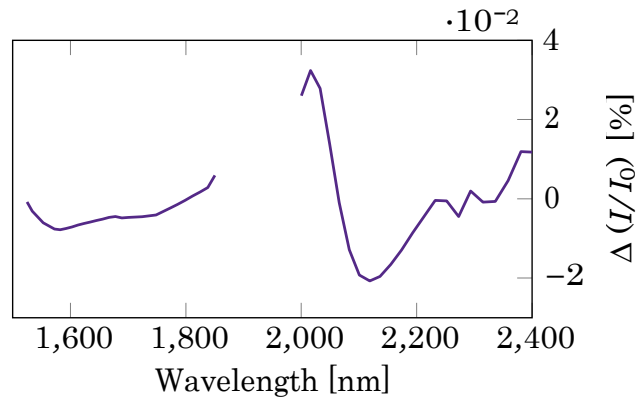

Figure 1: (a) Absorptivity of water and molar absorptivitity of glucose as tabulated. ${ }^{1}$ Note the difference in the orders of magnitude between water and glucose. (b) The difference in the intensity of $4 \mathrm{~mm}$ and $3 \mathrm{~mm}$ for $l=1 \mathrm{~mm}$.

In the original paper, we concluded that a system measuring glucose should be able to measure a difference corresponding to the highest possible percentage change in absorption, which was $0.003 \%$. However, we believe a more robust bound is the average absolute difference in absorbance across the range. For the updated difference presented in this erratum, the needed coefficient of variation (CV) for the first overtone band is therefore estimated to $0.0045 \%$ and a CV of $0.0117 \%$ is needed for the combination band. 
Later in the paper, we argued that the set limit could be relaxed according to $\sigma=\sigma_{0} / \overline{p / N_{v}}$, with $p$ channel variables that are transformed to $N_{v}$ latent variables. For $p=512$ and $N_{v}=5$, the limit of a CV of $0.0045 \%$ would increase by approximately a tenfold to $0.045 \%$ for the first overtone band, instead of $0.03 \%$ which was estimated in the original paper. The updated estimate is on the same order of magnitude and does not influence the conclusion.

\section{REFERENCES}

[1] A. K. Amerov, J. Chen and M. A. Arnold, "Molar Absorptivities of Glucose and other Biological Molecules in Aqueous Solutions over the First Overtone and Combination Regions of the Near-Infrared Spectrum," Appl. Spectrosc., 58(10), 1195-1204 (2004).

[2] L. Kou, D. Labrie and P. Chylek, "Refractive indices of water and ice in the $0.65 \mu \mathrm{m}$ to $2.5 \mu \mathrm{m}$ spectral range," Appl. Opt. 32, (1993).

Further author information and correspondence to S.S.F.: E-mail: silje.fuglerud@ntnu.no 